\title{
EDITORIAL
}

\section{Clinical learning environment and evidence-based nursing education}

In health sciences, the effort to implement research evidence is an important and integrating characteristic of research, education and clinical practice. We discuss practice and evidence-based health care but in Central and Eastern European countries, less attention is paid to evidence-based education.

Professional training of prospective health workers is a comprehensive educational trajectory during which time various ways of acquiring professional competence are promoted in students. Its irreplaceable and vital strategic section is clinical learning (accounting for approximately 50\% of curricula) integrating theory with practice. In Europe, research focused on the clinical environment in professional training of nurses and midwives has mainly been influenced by transformation of undergraduate nursing education that European Union countries have been continuously undergoing since the 1960s (Saarikoski et al., 2013). As part of multiple European projects, international studies (Warne et al., 2010; Antohe et al., 2016; Dobrowolska et al., 2016) compared the clinical environment with regard to organization of clinical learning among European countries. The first comparative studies were mainly performed in Western European countries (Warne et al., 2010). Following the research in Western Europe (Warne et al., 2010; Saarikoski et al., 2013), a comparative research project called Empowering the Professionalization of Nurses through Mentorship was conducted in Central and Eastern European countries (Czech Republic, Hungary, Romania and Lithuania) (Antohe et al., 2016). The results of the above studies underline the effectiveness of individualized approaches to guiding students in clinical training.

A considerable proportion of research studies are concerned with systematic monitoring of the effect of innovative teaching strategies (especially simulation technologies) and assessment methods (the focus being particularly on objective structured clinical examinations) on the development of students' competence (Cant, McKenna, Cooper, 2013). In multiple European countries (e.g. Finland or Great Britain), there has been a long-term trend to continuously monitor the clinical learning environment in health care settings and its impact on the development of key competences of prospective health workers. The global shortage of nurses and young nurses leaving the profession prematurely intensify the interest of European educational institutions in evidence-based education. Continuous auditing of clinical learning followed by innovations in clinical learning, strengthening the role of clinical training mentors and the individual mentor-student supervision relationship, implementing reflexive practice, developing methods for assessing students' competence are examples of strategies for increasing the effectiveness of the clinical environment and acquiring key nursing competences from several European countries. In the Czech Republic and Slovakia, nursing education and competences have, to a certain extent, become political issues with lively discussions on the advantages of the former system of nursing education at secondary medical schools. Such discussions, however, lack what we wanted to stress in the editorial - the evidence. In our setting, clinical learning was, until a certain moment, rather perceived as a formal component of health worker education with no detailed analysis of its quality and a standard mode of action that had not changed for a long time. Compared to traditional preclinical, theoretical teaching, however, clinical learning has its own specific requirements, determinants, anticipation and importance. And it is the significance of clinical learning in professional training of health workers that requires quality control and use of transparent, consistent and measurable indicators of the process, content and outcomes of clinical learning on the part of educational institutions.

Elena Gurková, RN, PhD Associate Professor

Department of Nursing, Faculty of Health Care University of Prešov in Prešov, Slovakia 


\section{References}

Antohe I, Riklikiene O, Tichelaar E, Saarikoski M. Clinical education and training of student nurses in four moderately new European Union countries: assessment of students' satisfaction with the learning environment. Nurse Education in Practice. 2016;17:139-144.

Cant R, McKenna L, Cooper S. Assessing preregistration nursing students' clinical competence: a systematic review of objective measures. International Journal of Nursing Practice. 2013;19(2):163-176.

Dobrowolska B, McGonagle I, Kane R, Jackson CS, Kegl B, Bergin M, Cabrera E, Cooney-Miner D, Di Cara V, Dimoski Z, Kekus D, Pajnkihar M, Prlić N, Sigurdardottir AK, Wells J, Palese A. Patterns of clinical mentorship in undergraduate nurse education: A comparative case analysis of eleven EU and non-EU countries. Nurse Education Today. 2016;36:44-52.

Saarikoski M, Kaila P, Lambrinou E, Pérez Cañaveras RM, Tichelaar E, Tomietto M, Warne T. Students' experiences of cooperation with nurse teacher during their clinical placements: an empirical study in a Western European context. Nurse Education in Practice. 2013;13(2):78-82.

Warne T, Johansson UB, Papastavrou E, Tichelaar E, Tomietto M, Van den Bossche K, Moreno MF, Saarikoski M. An exploration of the clinical learning experience of nursing students in nine European countries. Nurse Education Today. 2010;30(8):809-815. 